\title{
A PLANAR RANDOM MOTION WITH AN INFINITE NUMBER OF DIRECTIONS CONTROLLED BY THE DAMPED WAVE EQUATION
}

\author{
ALEXANDER D. KOLESNIK, ${ }^{*}$ Academy of Sciences of Moldova \\ ENZO ORSINGHER, ${ }^{* *}$ University of Rome 'La Sapienza'
}

\begin{abstract}
We consider the planar random motion of a particle that moves with constant finite speed $c$ and, at Poisson-distributed times, changes its direction $\theta$ with uniform law in $[0,2 \pi)$. This model represents the natural two-dimensional counterpart of the wellknown Goldstein-Kac telegraph process. For the particle's position $(X(t), Y(t)), t>0$, we obtain the explicit conditional distribution when the number of changes of direction is fixed. From this, we derive the explicit probability law $f(x, y, t)$ of $(X(t), Y(t))$ and show that the density $p(x, y, t)$ of its absolutely continuous component is the fundamental solution to the planar wave equation with damping. We also show that, under the usual Kac condition on the velocity $c$ and the intensity $\lambda$ of the Poisson process, the density $p$ tends to the transition density of planar Brownian motion. Some discussions concerning the probabilistic structure of wave diffusion with damping are presented and some applications of the model are sketched.
\end{abstract}

Keywords: Random motion; finite speed; uniformly distributed direction; telegraph process; damped wave equation; membrane vibration; Bessel function; planar Brownian motion; Sonine formula

2000 Mathematics Subject Classification: Primary 60K99

Secondary 62G30; 60K35; 60J60; 60H30

\section{Introduction}

Particles moving in liquids are driven by surface tension forces and move chaotically. At random instants, they take directions $v=(\cos \theta, \sin \theta)$, with $\theta$ uniformly distributed in $[0,2 \pi)$. This has been observed from the very first investigations of random motions by Robert Brown, and modelled thereafter by many researchers. It is well known that in classical Brownian motion both the speed of the particle and the rate of the changes of direction are assumed to be infinite. However, it has been remarked by many scientists that infinite-speed models are not suitable for the description of real processes evolving at low speed. For this reason, various alternative models based on the assumption of finiteness both of speed and of the rate of change of direction have been proposed.

The first probabilistic models of diffusion with finite speed of propagation appeared in the pioneering works of Goldstein (1951) and Kac (1974) (of which the latter is a reprinting of a 1956 paper). They studied the one-dimensional random motion of a particle moving

Received 3 March 2005; revision received 26 June 2005.

* Postal address: Institute of Mathematics and Computer Science, Academy of Sciences of Moldova, Academy Street 5 , Kishinev, MD-2028, Moldova. Email address: kolesnik@math.md

** Postal address: Dipartimento di Statistica, Probabilità e Statistiche Applicate, University of Rome 'La Sapienza', Piazzale Aldo Moro 5, 00185 Roma, Italy. Email address: enzo.orsingher@uniroma1.it 
forwards and backwards with constant speed $c$ and whose changes of direction are governed by a homogeneous Poisson process with rate $\lambda>0$.

It has been proved that the position $X(t), t>0$, of the particle performing this motion has distribution $p(x, t) \mathrm{d} x=\operatorname{Pr}\{X(t) \in \mathrm{d} x\}, x \in[-c t, c t]$, satisfying the following hyperbolic equation (which is a form of the so-called telegraph equation):

$$
\frac{\partial^{2} p}{\partial t^{2}}+2 \lambda \frac{\partial p}{\partial t}=c^{2} \frac{\partial^{2} p}{\partial x^{2}} .
$$

The density $p \equiv p(x, t)$ can be obtained by solving (1) with the initial conditions

$$
p(x, 0)=\delta(x), \quad p_{t}(x, 0)=0,
$$

where $\delta(x)$ is the Dirac delta-function and the subscript ' $t$ ' denotes partial differentiation with respect to time. In other words, the density $p(x, t)$ is the fundamental solution to the telegraph equation (1). The explicit form of the absolutely continuous part of the distribution was given by Orsingher (1990) and Pinsky (1991), and is expressed essentially as a combination of Bessel functions with imaginary arguments.

After the introduction of the telegraph process, many efforts were made to extend the onedimensional Goldstein-Kac model to multidimensional spaces. The main difficulty in doing so stems from the fact that, while on the line there are only two directions (i.e. forwards and backwards), in a multidimensional space there is a continuum of directions. In this case, the number of equations of the differential system for the transition densities also forms a continuum and, thus, the standard methods of obtaining the governing equations (substitutions, elimination, etc.) cannot be applied.

For this reason, the idea of discretization of the continuous spectrum of directions came to dominate in this field of research for many years. There were many papers devoted to similar models of motion on the plane with a finite number of possible directions changing randomly with different rules. In Orsingher (1986), a model of a particular finite-speed planar diffusive motion leading to the two-dimensional telegraph equation was presented. Motion in three directions has also been considered and the corresponding third-order governing partial differential equation obtained by Kolesnik (1989). A similar motion with three cyclically changing directions and with renewal processes representing the time lengths between successive changes was presented by Di Crescenzo (2002). A motion on the plane with four mutually orthogonal directions and reflection was investigated by Kolesnik and Orsingher (2002), and a planar model with four cyclically changing directions was studied by Leorato et al. (2003). The general case of symmetric planar random motion at finite speed (also called random evolution or a transport process) with an arbitrary, finite number of directions was studied by Kolesnik and Turbin (1998) and the related higher-order partial differential equation governing the distribution of the particle's position was obtained.

In the ensemble of works devoted to planar motion at finite speed, one should especially distinguish those (which are very few indeed) in which the explicit distributions were obtained. For the three-direction model with different types of change mechanism, the explicit distributions have recently been derived by Leorato and Orsingher (2004) by means of the order statistics approach and by applying some generalizations of Bose-Einstein statistics. In Orsingher (2000), the explicit distribution for motion with four mutually orthogonal directions without reflection was obtained. This distribution has the form of a fairly complex combination of products of Bessel functions with imaginary arguments, and their time derivatives. We 


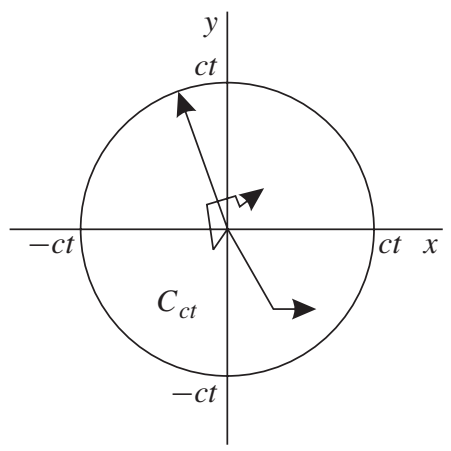

FIGURE 1: An example of three sample paths in which zero, one, and four changes of direction occur.

emphasize that the explicit derivation of the distribution is undoubtedly the most desirable aim of any research in this area.

In some specific contexts (random motion on computer screens, for example) the assumption that the directions are finite in number (and, in particular, orthogonal) seems to be the most convenient. However, the idea that at each change of direction the particle can choose any possible orientation is obviously closer to reality. This makes it necessary to study motion with a continuous number of directions. However, there exist only a few works in which such models are studied. In the monograph by Tolubinsky (1969, Chapter 2, pp. 35-60), the two- and three-dimensional random motion at finite speed was examined and the fundamental solution (a Green's function) of the governing integrodifferential system for the partial transition densities was obtained in a fairly complicated integral form. Models with uniformly distributed orientations for the steps made in the plane and in space were studied by Stadje (1987) (some details of his derivation are given in Section 3), Stadje (1989), and by Masoliver et al. (1993), and the explicit distributions for such motions obtained by means of recursive arguments.

However, as mentioned above, the problem of describing this type of random motion by means of partial differential equations has remained open for many decades. Moreover, it has stimulated intense discussion among researchers on whether such motion can be described by the multidimensional telegraph equation in a similar way to the one-dimensional GoldsteinKac telegraph process. Some researchers have simply replaced the operator $\partial^{2} / \partial x^{2}$ on the right-hand side of (1) with the multidimensional Laplacian. Other researchers considered that such a formal replacement was unjustified. Bartlett (1978, p. 705) wrote 'such equivalence is more doubtful in the multidimensional case'. Tolubinsky $(1969$, p. 49) has described such attempts as 'unjustified'.

The authors believe that the present paper answers the main question raised in the discussion, at least in the two-dimensional case. Here, we give a strict mathematical proof that planar random evolution with a continuous number of directions is governed by the two-dimensional telegraph equation, and the transition function of the motion is found explicitly. The authors also believe that the paper provides some insight into possible applications of the two-dimensional telegraph equation in applied models.

The planar motion considered here is that of a particle starting at the origin, moving with constant finite speed $c$, and taking uniformly distributed directions at Poisson times. The sample paths therefore appear to be continuous and composed of segments of random length and orientation $\theta$, which is uniformly distributed in $[0,2 \pi$ ) (see Figure 1). Note that the total length of each trajectory at any instant $t>0$ is $c t$. 
The set of possible positions of the particle at time $t>0$ is a disc $C_{c t}$ of radius $c t$, and the distribution of the position $f \equiv f(x, y, t)$ has a singular component concentrated on the circumference $\partial C_{c t}$ (corresponding to the case in which the initial direction is maintained until time $t$ ). When the number of changes of direction is greater than or equal to one, the particle is located inside $C_{c t}$ and, since the trajectories are continuous and the motion is of finite speed, the distribution has an absolutely continuous part.

Our main result is that the absolutely continuous part $p \equiv p(x, y, t)$ of $f$ has the succinct analytical form

$$
p(x, y, t)=\frac{\lambda}{2 \pi c} \frac{\exp \left(-\lambda t+(\lambda / c) \sqrt{c^{2} t^{2}-\left(x^{2}+y^{2}\right)}\right)}{\sqrt{c^{2} t^{2}-\left(x^{2}+y^{2}\right)}}, \quad(x, y) \in \operatorname{int} C_{c t}, t>0,
$$

which, surprisingly, is simpler than its one-dimensional counterpart (see Orsingher (1990, Theorem 1), or Pinsky (1991, p. 9) for comparison).

Remarkably, the probability density (2) is the fundamental solution to the two-dimensional telegraph equation

$$
\frac{\partial^{2} p}{\partial t^{2}}+2 \lambda \frac{\partial p}{\partial t}=c^{2}\left\{\frac{\partial^{2} p}{\partial x^{2}}+\frac{\partial^{2} p}{\partial y^{2}}\right\}
$$

also called the planar damped wave equation.

We also obtain the following conditional distributions for the position $(X(t), Y(t)), t>0$, where $N(t)$ is the number of Poisson events that have occurred by time $t$ :

$$
\begin{array}{r}
\operatorname{Pr}\{X(t) \in \mathrm{d} x, Y(t) \in \mathrm{d} y \mid N(t)=n\}=\frac{n}{2 \pi(c t)^{n}}\left[c^{2} t^{2}-\left(x^{2}+y^{2}\right)\right]^{n / 2-1} \mathrm{~d} x \mathrm{~d} y, \\
n \geq 1,(x, y) \in \operatorname{int} C_{c t} .
\end{array}
$$

The function (4) is circularly symmetric (in the ( $x, y$ )-plane) for all $n \geq 1$ due to the uniform distribution of the choice of direction at each Poisson instant.

For $n=1$, we have the special and interesting distribution

$$
\begin{array}{r}
\operatorname{Pr}\{X(t) \in \mathrm{d} x, Y(t) \in \mathrm{d} y \mid N(t)=1\}=\frac{1}{2 \pi c t}\left[c^{2} t^{2}-\left(x^{2}+y^{2}\right)\right]^{-1 / 2} \mathrm{~d} x \mathrm{~d} y, \\
(x, y) \in \operatorname{int} C_{c t},
\end{array}
$$

which coincides with the Green's function of the planar wave equation (without damping), i.e. with the solution to the Cauchy problem

$$
\frac{\partial^{2} u}{\partial t^{2}}=c^{2}\left\{\frac{\partial^{2} u}{\partial x^{2}}+\frac{\partial^{2} u}{\partial y^{2}}\right\}, \quad u(x, y, 0)=\delta(x, y), \quad u_{t}(x, y, 0)=0,
$$

where $\delta(x, y)$ is the Dirac delta-function on $\mathbb{R}^{2}$. This means that the Green's function (5) can be obtained as the sum of two random, uniformly oriented displacements of respective lengths $c s$ and $c(t-s), s$ being a uniformly distributed random variable in $(0, t)$.

When $n=2$, the conditional distribution (4) becomes uniform in the disc $C_{c t}$, which was not expected. For $n \geq 3$, the distribution (4) takes a bell-shaped form due to the fact that, the more frequently do changes of the direction of motion occur, the more fragmented become the sample paths and the shorter becomes the distance of the moving particle from the origin. 
We also show that, under the Kac condition

$$
c \rightarrow \infty, \quad \lambda \rightarrow \infty, \quad \frac{c^{2}}{\lambda} \rightarrow 1,
$$

the telegraph equation (3) becomes the planar heat equation and the density (2) tends to the transition density of the planar standard Brownian motion, as happens in the one-dimensional case (see Kac (1974), Orsingher (1990), or Brooks (1999)).

The density (2) takes an especially interesting form in polar coordinates, namely

$$
\widetilde{p}(\rho, \theta, t)=\frac{\lambda}{2 \pi c} \frac{\rho \exp \left(-\lambda t+(\lambda / c) \sqrt{c^{2} t^{2}-\rho^{2}}\right)}{\sqrt{c^{2} t^{2}-\rho^{2}}}, \quad 0<\rho<c t, 0 \leq \theta<2 \pi, t>0,
$$

which shows that the radial component is independent of the angular one. The situation suggested by (7) is that of the Brownian transition function in polar coordinates, where the radial and angular components are also independent.

Our results generalize those obtained by Stadje (1987) and by Masoliver et al. (1993) for the case of arbitrary, finite speed $c$, which is very important in studying the limiting behaviour of the motion. We emphasize that, for $c=1$, the densities (2) and (4) respectively coincide with those given in formulae (1.3) and (2.1) of Stadje (1987). The method of derivation differs from those of previous results. We also present different explicit forms for the characteristic functions (and the proof of how they can be derived from one another); in Stadje (1987), these results were given only implicitly.

The motion studied and the results obtained here have a straightforward mechanical interpretation. Equation (3) emerges in the context of damped vibrations of membranes. The damping forces are indicated by the presence of a first-order time derivative in (3). Forces playing the role of obstacles cause sudden deviations in the motion of the particles constituting the vibrating medium. Equation (2) can be interpreted similarly. The factor $\left(c^{2} t^{2}-\left(x^{2}+y^{2}\right)\right)^{-1 / 2}$ represents an outgoing plane wave and the exponential numerator introduces the damping effect.

The authors believe that the motion presented and analysed here is a model applicable to many diffusion processes with finite speed, occurring, for example, in hydrodynamics, biology, and various forms of planar random vibration.

\section{Description of motion and conditional distributions}

We consider a particle starting its motion from the origin $x=y=0$ of the plane $\mathbb{R}^{2}$ at time $t=0$. The particle has constant, finite speed $c$. The initial direction is a random variable $\theta$ uniformly distributed in $[0,2 \pi)$. The particle changes direction at random instants that form a homogeneous Poisson process of rate $\lambda>0$. At these moments, it instantaneously takes on a new direction with uniform distribution in $[0,2 \pi)$, independently of its previous direction.

Let $(X(t), Y(t)), t>0$, be the position of the particle at an arbitrary time $t$. In this section, we concentrate our attention on the conditional distributions

$$
\operatorname{Pr}\{X(t) \in \mathrm{d} x, Y(t) \in \mathrm{d} y \mid N(t)=n\}, \quad n \geq 1,
$$

where $N(t)$ is the number of Poisson events in the interval $(0, t)$.

At any time $t>0$, with probability 1 the particle is located in the disc

$$
C_{c t}=\left\{(x, y) \in \mathbb{R}^{2}: x^{2}+y^{2} \leq c^{2} t^{2}\right\}
$$


of radius $c t$. If no Poisson event occurs, the particle lies on the circumference

$$
\partial C_{c t}=\left\{(x, y) \in \mathbb{R}^{2}: x^{2}+y^{2}=c^{2} t^{2}\right\}
$$

the probability of this event is

$$
\operatorname{Pr}\left\{(X(t), Y(t)) \in \partial C_{c t}\right\}=\mathrm{e}^{-\lambda t} .
$$

The remaining part of the distribution is concentrated in the interior of the disc (9) and forms the absolutely continuous part of

$$
\operatorname{Pr}\{X(t) \in \mathrm{d} x, Y(t) \in \mathrm{d} y\}, \quad(x, y) \in \operatorname{int} C_{c t} .
$$

Therefore, a density $p=p(x, y, t),(x, y) \in$ int $C_{c t}, t>0$, exists for (10). This density is the main focus of our research. Our first result concerns the explicit form of the conditional distributions (8).

Theorem 1. For all $n \geq 1$ and any $t>0$, we have

$$
\begin{array}{r}
\operatorname{Pr}\{X(t) \in \mathrm{d} x, Y(t) \in \mathrm{d} y \mid N(t)=n\}=\frac{n}{2 \pi(c t)^{n}}\left[c^{2} t^{2}-\left(x^{2}+y^{2}\right)\right]^{n / 2-1} \mathrm{~d} x \mathrm{~d} y, \\
(x, y) \in \operatorname{int} C_{c t} .
\end{array}
$$

Proof. If $N(t)=n$, the displacement of the particle at any time $t>0$ is given by

$$
X(t)=c \sum_{j=1}^{n+1}\left(s_{j}-s_{j-1}\right) \cos \theta_{j}, \quad Y(t)=c \sum_{j=1}^{n+1}\left(s_{j}-s_{j-1}\right) \sin \theta_{j},
$$

where $\theta_{j}, j=1, \ldots, n+1$, are independent random variables uniformly distributed in $[0,2 \pi)$, the $s_{j}, j=1, \ldots, n$, represent the instants at which Poisson events occur, and $s_{0}=0$ and $s_{n+1}=t$.

We now evaluate the conditional characteristic function of (12):

$$
\begin{aligned}
I_{n} & =\mathrm{E}\{\exp (\mathrm{i} \alpha X(t)+\mathrm{i} \beta Y(t)) \mid N(t)=n\} \\
& =\mathrm{E}\left\{\exp \left(\mathrm{i} \alpha c \sum_{j=1}^{n+1}\left(s_{j}-s_{j-1}\right) \cos \theta_{j}+\mathrm{i} \beta c \sum_{j=1}^{n+1}\left(s_{j}-s_{j-1}\right) \sin \theta_{j}\right)\right\} \\
& =\frac{n !}{t^{n}} \int_{0}^{t} \mathrm{~d} s_{1} \int_{s_{1}}^{t} \mathrm{~d} s_{2} \cdots \int_{s_{n-1}}^{t} \mathrm{~d} s_{n}\left\{\frac{1}{(2 \pi)^{n+1}} \int_{0}^{2 \pi} \mathrm{d} \theta_{1} \int_{0}^{2 \pi} \mathrm{d} \theta_{2} \cdots \int_{0}^{2 \pi} \mathrm{d} \theta_{n+1}\right. \\
& \left.\times \frac{n !}{t^{n}} \int_{0}^{t} \mathrm{~d} s_{1} \int_{s_{1}}^{t} \mathrm{~d} s_{2} \cdots \int_{s_{n-1}}^{t} \mathrm{~d} s_{n}\left(\mathrm{i} c \sum_{j=1}^{n+1}\left(s_{j}-s_{j-1}\right)\left(\alpha \cos \theta_{j}+\beta \sin \theta_{j}\right)\right)\right\} \\
& \times \prod_{j=1}^{n+1}\left[\frac{1}{2 \pi} \int_{0}^{2 \pi} \exp \left(\mathrm{i} c\left(s_{j}-s_{j-1}\right)\left(\alpha \cos \theta_{j}+\beta \sin \theta_{j}\right)\right) \mathrm{d} \theta_{j}\right] \\
& =\frac{n !}{t^{n}} \int_{0}^{t} \mathrm{~d} s_{1} \int_{s_{1}}^{t} \mathrm{~d} s_{2} \cdots \int_{s_{n-1}}^{t} \mathrm{~d} s_{n}\left\{\prod_{j=1}^{n+1} J_{0}\left(c\left(s_{j}-s_{j-1}\right) \sqrt{\alpha^{2}+\beta^{2}}\right)\right\} .
\end{aligned}
$$


In the last step, we have used the well-known integral representation of the Bessel function,

$$
J_{0}\left(x \sqrt{a^{2}+b^{2}}\right)=\frac{1}{2 \pi} \int_{0}^{2 \pi} \exp (\mathrm{i} x(a \cos \theta+b \sin \theta)) \mathrm{d} \theta .
$$

It is surprising that the $n$-fold complex integral (13) can be evaluated explicitly. We note that, upon application of Formula 6.581(3) of Gradshteyn and Ryzhik (1980), namely

$$
\begin{array}{r}
\int_{0}^{a} x^{\mu}(a-x)^{v} J_{\mu}(x) J_{v}(a-x) \mathrm{d} x=\frac{\Gamma\left(\mu+\frac{1}{2}\right) \Gamma\left(v+\frac{1}{2}\right)}{\sqrt{2 \pi} \Gamma(\mu+v+1)} a^{\mu+v+1 / 2} J_{\mu+v+1 / 2}(a), \\
\operatorname{Re} \mu>-\frac{1}{2}, \operatorname{Re} v>-\frac{1}{2},
\end{array}
$$

the integral with respect to $s_{n}$, after some elementary substitutions, becomes

$$
\begin{aligned}
\int_{s_{n-1}}^{t} & J_{0}\left(c\left(s_{n}-s_{n-1}\right) \sqrt{\alpha^{2}+\beta^{2}}\right) J_{0}\left(c\left(t-s_{n}\right) \sqrt{\alpha^{2}+\beta^{2}}\right) \mathrm{d} s_{n} \\
= & \frac{\Gamma\left(\frac{1}{2}\right) \Gamma\left(\frac{1}{2}\right)}{\sqrt{2 \pi} \Gamma(1)}\left(\frac{t-s_{n-1}}{c \sqrt{\alpha^{2}+\beta^{2}}}\right)^{1 / 2} J_{1 / 2}\left(c\left(t-s_{n-1}\right) \sqrt{\alpha^{2}+\beta^{2}}\right) .
\end{aligned}
$$

By applying the same formula of Gradshteyn and Ryzhik, we can write the integral with respect to $s_{n-1}$ as

$$
\begin{aligned}
\frac{\Gamma\left(\frac{1}{2}\right) \Gamma\left(\frac{1}{2}\right)}{\sqrt{2 \pi} \Gamma(1)} \int_{s_{n-2}}^{t}\left(\frac{t-s_{n-1}}{c \sqrt{\alpha^{2}+\beta^{2}}}\right)^{1 / 2} J_{1 / 2}\left(c\left(t-s_{n-1}\right) \sqrt{\alpha^{2}+\beta^{2}}\right) \\
\times J_{0}\left(c\left(s_{n-1}-s_{n-2}\right) \sqrt{\alpha^{2}+\beta^{2}}\right) \mathrm{d} s_{n-1} \\
=\frac{\Gamma\left(\frac{1}{2}\right) \Gamma\left(\frac{1}{2}\right)}{\sqrt{2 \pi} \Gamma(1)} \frac{\Gamma\left(\frac{1}{2}\right) \Gamma(1)}{\sqrt{2 \pi} \Gamma\left(\frac{3}{2}\right)}\left(\frac{t-s_{n-2}}{c \sqrt{\alpha^{2}+\beta^{2}}}\right) J_{1}\left(c\left(t-s_{n-2}\right) \sqrt{\alpha^{2}+\beta^{2}}\right) .
\end{aligned}
$$

After the $m$ th, $m \leq n-1$, integration, by the same procedure we can write $I_{n}$ in the form

$$
\begin{aligned}
I_{n}=\frac{n !}{t^{n}} & \prod_{k=1}^{m} \frac{\Gamma\left(\frac{1}{2}\right) \Gamma\left(\frac{1}{2} k\right)}{\sqrt{2 \pi} \Gamma\left(\frac{1}{2}(k+1)\right)} \int_{0}^{t} \mathrm{~d} s_{1} \cdots \int_{s_{n-m-2}}^{t} \mathrm{~d} s_{n-m-1} \\
& \times\left[\int_{s_{n-m-1}}^{t}\left(\frac{t-s_{n-m}}{c \sqrt{\alpha^{2}+\beta^{2}}}\right)^{m / 2} J_{m / 2}\left(c\left(t-s_{n-m}\right) \sqrt{\alpha^{2}+\beta^{2}}\right)\right. \\
& \left.\times J_{0}\left(c\left(s_{n-m}-s_{n-m-1}\right) \sqrt{\alpha^{2}+\beta^{2}}\right) \mathrm{d} s_{n-m}\right] \\
& \times J_{0}\left(c\left(s_{n-m-1}-s_{n-m-2}\right) \sqrt{\alpha^{2}+\beta^{2}}\right) \cdots J_{0}\left(c s_{1} \sqrt{\alpha^{2}+\beta^{2}}\right) .
\end{aligned}
$$

By inspecting formulae (15), (16), and (17), we see that a systematic rule in the results of the integrations becomes apparent. At the $(n-1)$ th stage of the integration process, we obtain

$$
\begin{aligned}
I_{n}=\frac{n !}{t^{n}} \prod_{k=1}^{n-1} \frac{\Gamma\left(\frac{1}{2}\right) \Gamma\left(\frac{1}{2} k\right)}{\sqrt{2 \pi} \Gamma\left(\frac{1}{2}(k+1)\right)} \int_{0}^{t} & \left(\frac{t-s_{1}}{c \sqrt{\alpha^{2}+\beta^{2}}}\right)^{(n-1) / 2} \\
& \times J_{(n-1) / 2}\left(c\left(t-s_{1}\right) \sqrt{\alpha^{2}+\beta^{2}}\right) J_{0}\left(c s_{1} \sqrt{\alpha^{2}+\beta^{2}}\right) \mathrm{d} s_{1} .
\end{aligned}
$$


By applying Formula 6.581(3) of Gradshteyn and Ryzhik (1980) once more, and noting that

$$
n ! \prod_{k=1}^{n} \frac{\Gamma\left(\frac{1}{2}\right) \Gamma\left(\frac{1}{2} k\right)}{\sqrt{2 \pi} \Gamma\left(\frac{1}{2}(k+1)\right)}=2^{n / 2} \Gamma\left(\frac{n}{2}+1\right),
$$

we obtain the conditional characteristic function

$$
\mathrm{E}\{\exp (\mathrm{i} \alpha X(t)+\mathrm{i} \beta Y(t)) \mid N(t)=n\}=\frac{2^{n / 2} \Gamma\left(\frac{1}{2} n+1\right)}{\left(c t \sqrt{\alpha^{2}+\beta^{2}}\right)^{n / 2}} J_{n / 2}\left(c t \sqrt{\alpha^{2}+\beta^{2}}\right), \quad n \geq 1 .
$$

This expression can be inverted directly, but this involves complicated calculations involving hypergeometric series. Instead, we prefer to calculate the Fourier transform of (11) and show that it coincides with (18).

By using polar coordinates and applying (14) we find that

$$
\begin{aligned}
& \iint_{C_{c t}} \mathrm{e}^{\mathrm{i} \alpha x+\mathrm{i} \beta y} \operatorname{Pr}\{X(t) \in \mathrm{d} x, Y(t) \in \mathrm{d} y \mid N(t)=n\} \\
& =\iint_{C_{c t}} \mathrm{e}^{\mathrm{i} \alpha x+\mathrm{i} \beta y} \frac{n}{2 \pi(c t)^{n}}\left[c^{2} t^{2}-\left(x^{2}+y^{2}\right)\right]^{n / 2-1} \mathrm{~d} x \mathrm{~d} y \\
& =\frac{n}{2 \pi(c t)^{n}} \int_{0}^{2 \pi} \int_{0}^{c t} \exp (\mathrm{i} \rho(\alpha \cos \theta+\beta \sin \theta))\left(c^{2} t^{2}-\rho^{2}\right)^{n / 2-1} \rho \mathrm{d} \rho \mathrm{d} \theta \\
& =\frac{n}{(c t)^{n}} \int_{0}^{c t} \rho\left(c^{2} t^{2}-\rho^{2}\right)^{n / 2-1} J_{0}\left(\rho \sqrt{\alpha^{2}+\beta^{2}}\right) \mathrm{d} \rho \\
& =n \int_{0}^{1} w\left(1-w^{2}\right)^{n / 2-1} J_{0}\left(c t w \sqrt{\alpha^{2}+\beta^{2}}\right) \mathrm{d} w \\
& =n \sum_{m=0}^{\infty} \frac{(-1)^{m}}{(m !)^{2}}\left(\frac{c t \sqrt{\alpha^{2}+\beta^{2}}}{2}\right)^{2 m} \int_{0}^{1} w^{2 m+1}\left(1-w^{2}\right)^{n / 2-1} \mathrm{~d} w \\
& =\frac{n}{2} \sum_{m=0}^{\infty} \frac{(-1)^{m}}{(m !)^{2}}\left(\frac{c t \sqrt{\alpha^{2}+\beta^{2}}}{2}\right)^{2 m} \int_{0}^{1} z^{m}(1-z)^{n / 2-1} \mathrm{~d} z \\
& =\frac{n}{2} \sum_{m=0}^{\infty} \frac{(-1)^{m}}{(m !)^{2}}\left(\frac{c t \sqrt{\alpha^{2}+\beta^{2}}}{2}\right)^{2 m} \frac{\Gamma(m+1) \Gamma\left(\frac{1}{2} n\right)}{\Gamma\left(m+\frac{1}{2} n+1\right)} \\
& =\Gamma\left(\frac{n}{2}+1\right) \sum_{m=0}^{\infty} \frac{(-1)^{m}}{m !}\left(\frac{c t \sqrt{\alpha^{2}+\beta^{2}}}{2}\right)^{2 m+n / 2} \frac{1}{\Gamma\left(m+\frac{1}{2} n+1\right)}\left(\frac{2}{c t \sqrt{\alpha^{2}+\beta^{2}}}\right)^{n / 2} \\
& =\frac{2^{n / 2} \Gamma\left(\frac{1}{2} n+1\right)}{\left(c t \sqrt{\alpha^{2}+\beta^{2}}\right)^{n / 2}} J_{n / 2}\left(c t \sqrt{\alpha^{2}+\beta^{2}}\right),
\end{aligned}
$$

which coincides with (18). The theorem is thus proved.

Remark 1. Let us examine some specific particular cases of the distribution (11). For $n=1$, the conditional density (11) becomes

$$
\operatorname{Pr}\{X(t) \in \mathrm{d} x, Y(t) \in \mathrm{d} y \mid N(t)=1\}=\frac{1}{2 \pi c t} \frac{\mathrm{d} x \mathrm{~d} y}{\sqrt{c^{2} t^{2}-\left(x^{2}+y^{2}\right)}}, \quad(x, y) \in \operatorname{int} C_{c t},
$$


which is the Green's function of planar waves without damping. It represents how a wave starting at the origin moves outwards. The wavefront is infinitely high near $\partial C_{c t}$ and possesses a nonvanishing real tail. However, despite the fact that (19) has an infinite discontinuity on the boundary $\partial C_{c t}$, we can easily check that

$$
\lim _{\varepsilon \rightarrow 0} \frac{1}{c t} \int_{c t-\varepsilon}^{c t} \frac{\rho \mathrm{d} \rho}{\sqrt{c^{2} t^{2}-\rho^{2}}}=0
$$

and, therefore, the density (19) is integrable in $C_{c t}$. From our analysis, it can be seen that this important function can be obtained as the distribution of two random displacements, ( $\left.c s \cos \theta_{1}, c s \sin \theta_{1}\right)$ and $\left(c(t-s) \cos \theta_{2}, c(t-s) \sin \theta_{2}\right)$, with $\theta_{1}$ and $\theta_{2}$ uniform in $[0,2 \pi)$ and $s$ uniform in $(0, t)$. This also means that if only one change of direction occurs, then the density near the border is large.

For $n=2$, (11) unexpectedly gives the uniform density on $C_{c t}$ :

$$
\operatorname{Pr}\{X(t) \in \mathrm{d} x, Y(t) \in \mathrm{d} y \mid N(t)=2\}=\frac{\mathrm{d} x \mathrm{~d} y}{\pi c^{2} t^{2}}, \quad(x, y) \in \operatorname{int} C_{c t} .
$$

This means that after two changes of direction the particle is located near the border of $C_{c t}$ or near the origin with the same probability. In a certain sense, the third displacement somehow cancels the 'outward push' noted after the first change of direction.

For $n \geq 3$, the form of the conditional distribution does not change in such a drastic manner. Rather, it takes on a bell-shaped structure: for example, for $n=3$ we have

$$
\operatorname{Pr}\{X(t) \in \mathrm{d} x, Y(t) \in \mathrm{d} y \mid N(t)=3\}=\frac{3 \mathrm{~d} x \mathrm{~d} y}{2 \pi(c t)^{3}} \sqrt{c^{2} t^{2}-\left(x^{2}+y^{2}\right)}, \quad(x, y) \in \operatorname{int} C_{c t} .
$$

The reason for the appearance of the bell form is that, when a sufficient number of changes of direction has been recorded (the minimal number clearly being three), the sample paths are so fragmented that the particle can barely leave the neighbourhood of the origin. In the one-dimensional case mentioned in the introduction, we know that for the telegraph process the conditional distribution is uniform when $N(t)=1$ and that bell-shaped densities appear for $n \geq 2$.

\section{The density of motion and its connection to the planar damped wave equation}

From Theorem 1, we can straightforwardly infer our main result.

Theorem 2. The absolutely continuous part of the distribution of $(X(t), Y(t)), t>0$, is given by

$$
\begin{array}{r}
\operatorname{Pr}\{X(t) \in \mathrm{d} x, Y(t) \in \mathrm{d} y\}=\frac{\lambda}{2 \pi c} \frac{\exp \left(-\lambda t+(\lambda / c) \sqrt{c^{2} t^{2}-\left(x^{2}+y^{2}\right)}\right)}{\sqrt{c^{2} t^{2}-\left(x^{2}+y^{2}\right)}} \mathrm{d} x \mathrm{~d} y, \\
(x, y) \in \operatorname{int} C_{c t}, t>0 .
\end{array}
$$

Proof. In view of Theorem 1, we have

$$
\begin{aligned}
\operatorname{Pr}\{X(t) \in \mathrm{d} x, Y(t) \in \mathrm{d} y\} & =\sum_{n=1}^{\infty} \operatorname{Pr}\{X(t) \in \mathrm{d} x, Y(t) \in \mathrm{d} y \mid N(t)=n\} \operatorname{Pr}\{N(t)=n\} \\
& =\frac{\mathrm{e}^{-\lambda t}}{2 \pi} \sum_{n=1}^{\infty} \frac{(\lambda t)^{n}}{n !} \frac{n}{(c t)^{n}}\left(c^{2} t^{2}-\left(x^{2}+y^{2}\right)\right)^{n / 2-1} \mathrm{~d} x \mathrm{~d} y,
\end{aligned}
$$

and after some elementary calculations we recover (20). 
We can also easily check that, for any $t>0$,

$$
\begin{aligned}
\iint_{C_{c t}} \operatorname{Pr}\{X(t) \in \mathrm{d} x, Y(t) \in \mathrm{d} y\} & =\frac{\lambda \mathrm{e}^{-\lambda t}}{2 \pi c} \int_{0}^{2 \pi} \mathrm{d} \theta \int_{0}^{c t} \frac{\exp \left((\lambda / c) \sqrt{c^{2} t^{2}-\rho^{2}}\right)}{\sqrt{c^{2} t^{2}-\rho^{2}}} \rho \mathrm{d} \rho \\
& =1-\mathrm{e}^{-\lambda t},
\end{aligned}
$$

where the missing part of the probability (namely $\mathrm{e}^{-\lambda t}$ ) pertains to the singular part of the distribution and is concentrated on $\partial C_{c t}$. The theorem is thus proved.

The shape of the density (20) is represented in Figure 2.

It is interesting to note the connections of our results to those already known. Stadje (1987) found the conditional distributions (11) in the case $c=1$ by means of a recursive argument that can be described as follows. Let $D(t)$ be the distance of the moving particle from the origin at time $t$ and let $D_{1}$ be its distance from the origin at the instant of the first Poisson event. Let $\theta_{1}$ denote the initial direction of motion. It is clear that

$$
\begin{aligned}
& \operatorname{Pr}\{D(t) \in \mathrm{d} \rho \mid N(t)=n\}=\int_{0}^{t} \int_{0}^{2 \pi} \operatorname{Pr}\left\{D(t) \in \mathrm{d} \rho, D_{1} \in \mathrm{d} s, \theta_{1} \in \mathrm{d} \alpha \mid N(t)=n\right\} \\
&= \int_{0}^{t} \int_{0}^{2 \pi} \operatorname{Pr}\left\{D_{1} \in \mathrm{d} s, \theta_{1} \in \mathrm{d} \alpha \mid N(t)=n\right\} \\
&= \times \operatorname{Pr}\left\{D(t) \in \mathrm{d} \rho \mid D_{1}=s, \theta_{1}=\alpha, N(t)=n\right\} \\
&=\int_{0}^{t} \int_{0}^{2 \pi} \operatorname{Pr}\left\{D_{1} \in \mathrm{d} s, \theta_{1} \in \mathrm{d} \alpha \mid N(t)=n\right\} \\
& \quad \times \operatorname{Pr}\{D(t-s) \in \mathrm{d}(\rho-s) \mid N(t)=n-1\} .
\end{aligned}
$$

In the last step, we have assumed that the motion starts from scratch at the position reached at the first Poisson event (i.e. it begins again with the same rules), and $\rho-s$ indicates the length of the difference between the vectors $(\rho, 0)$ and $(s, \alpha)$.

Furthermore,

$$
\begin{aligned}
\operatorname{Pr}\left\{D_{1} \in \mathrm{d} s, \theta_{1} \in \mathrm{d} \alpha \mid N(t)=n\right\} & =\frac{\operatorname{Pr}\left\{N(t)=n \mid D_{1} \in \mathrm{d} s, \theta_{1} \in \mathrm{d} \alpha\right\} \operatorname{Pr}\left\{D_{1} \in \mathrm{d} s, \theta_{1} \in \mathrm{d} \alpha\right\}}{\operatorname{Pr}\{N(t)=n\}} \\
& =\frac{\lambda \mathrm{e}^{-\lambda s}}{2 \pi} \frac{\operatorname{Pr}\{N(t-s)=n-1\}}{\operatorname{Pr}\{N(t)=n\}} \mathrm{d} s \mathrm{~d} \alpha \\
& =\frac{n}{2 \pi t}\left(\frac{t-s}{t}\right)^{n-1} \mathrm{~d} s \mathrm{~d} \alpha .
\end{aligned}
$$

If $D_{1}=s$ and $\theta_{1}=\alpha$ then at time $s$ the first event has occurred, and the other $n-1$ Poisson events must fall in the subsequent interval $(s, t)$. By substituting this last expression into the previous one, we obtain the formula on which the derivation in Stadje (1987) is based. The derivation of Masoliver et al. (1993) is also based on recursive arguments. They found and inverted the Fourier-Laplace transform.

Remark 2. From (20), it is easy to obtain

$$
\operatorname{Pr}\left\{(X(t), Y(t)) \in C_{r}\right\}=1-\exp \left(-\lambda t+\frac{\lambda}{c} \sqrt{c^{2} t^{2}-r^{2}}\right), \quad 0 \leq r<c t,
$$

which is the probability of the particle lying within any disc $C_{r}$ of radius $r, 0 \leq r<c t$. 


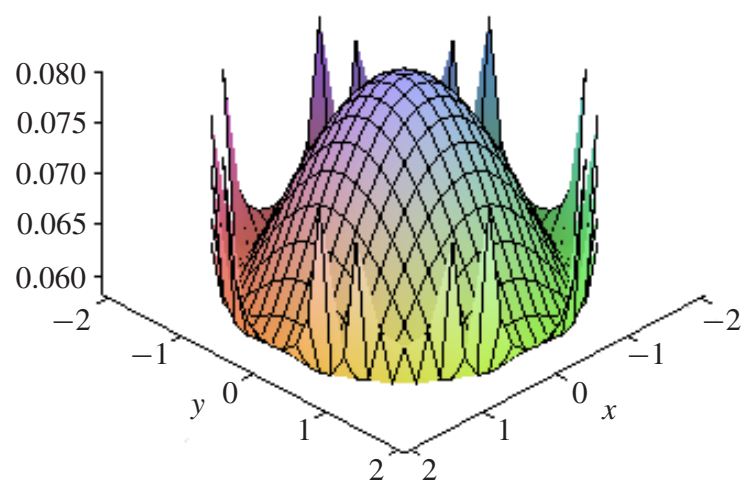

FIGURE 2: The shape of the density $p(x, y, t)$ at the instant $t=2$ (for $c=1$ and $\lambda=1$ ).

Remark 3. Density (20) is the product of the Green's function of planar waves (related to a single change of direction) and an exponential factor representing the damping effect produced by many changes of direction. Our model gives some insight into the mechanism of damped wave propagation. The forces acting on a vibrating membrane can be imagined as the superposition of random motions developing as zigzag lines, in contrast to the outward motion of the wave. The effect of these random motions is to hinder the energy propagation. The more changes of direction there are, the lower is the energy that spreads outward and, therefore, the smaller its contribution to wave propagation. The same damping effect can be observed in the case of one-dimensional waves where, surprisingly, the form of the distribution density is much more complicated than (20).

Remark 4. The complete density $f(x, y, t),(x, y) \in C_{c t}, t \geq 0$, of the distribution of $(X(t), Y(t))$ can be written in terms of generalized functions as follows, where $\delta(\cdot)$ is the Dirac delta-function and $\Theta(\cdot)$ is the Heaviside function:

$$
\begin{aligned}
f(x, y, t)= & \frac{\mathrm{e}^{-\lambda t}}{2 \pi c t} \delta\left(c^{2} t^{2}-x^{2}-y^{2}\right) \\
& +\frac{\lambda}{2 \pi c} \frac{\exp \left(-\lambda t+(\lambda / c) \sqrt{c^{2} t^{2}-\left(x^{2}+y^{2}\right)}\right)}{\sqrt{c^{2} t^{2}-\left(x^{2}+y^{2}\right)}} \Theta\left(c t-\sqrt{x^{2}+y^{2}}\right) .
\end{aligned}
$$

Remark 5. From (18) and by noting that

$$
\mathrm{E}\{\exp (\mathrm{i} \alpha X(t)+\mathrm{i} \beta Y(t)) \mid N(t)=0\}=J_{0}\left(c t \sqrt{\alpha^{2}+\beta^{2}}\right)
$$

we obtain the following characteristic function for the random vector $(X(t), Y(t))$ :

$$
\begin{aligned}
H(\alpha, \beta, t) & =\mathrm{E}\{\exp (\mathrm{i} \alpha X(t)+\mathrm{i} \beta Y(t))\} \\
& =\mathrm{e}^{-\lambda t} \sum_{n=0}^{\infty} \frac{(\lambda t)^{n}}{n !} \frac{2^{n / 2} \Gamma\left(\frac{1}{2} n+1\right)}{\left(c t \sqrt{\alpha^{2}+\beta^{2}}\right)^{n / 2}} J_{n / 2}\left(c t \sqrt{\alpha^{2}+\beta^{2}}\right)
\end{aligned}
$$


The characteristic function can also be expressed in integral form. By using polar coordinates, we can write

$$
\begin{aligned}
H(\alpha, \beta, t)= & \mathrm{E}\{\exp (\mathrm{i} \alpha X(t)+\mathrm{i} \beta Y(t))\} \\
= & \frac{\lambda \mathrm{e}^{-\lambda t}}{2 \pi c} \iint_{C_{c t}} \mathrm{e}^{\mathrm{i} \alpha x+\mathrm{i} \beta y} \frac{\exp \left((\lambda / c) \sqrt{c^{2} t^{2}-\left(x^{2}+y^{2}\right)}\right)}{\sqrt{c^{2} t^{2}-\left(x^{2}+y^{2}\right)}} \mathrm{d} x \mathrm{~d} y \\
& +\mathrm{e}^{-\lambda t} J_{0}\left(c t \sqrt{\alpha^{2}+\beta^{2}}\right) \\
= & \frac{\lambda \mathrm{e}^{-\lambda t}}{c} \int_{0}^{c t} J_{0}\left(\rho \sqrt{\alpha^{2}+\beta^{2}}\right) \frac{\exp \left((\lambda / c) \sqrt{c^{2} t^{2}-\rho^{2}}\right)}{\sqrt{c^{2} t^{2}-\rho^{2}}} \rho \mathrm{d} \rho \\
& +\mathrm{e}^{-\lambda t} J_{0}\left(c t \sqrt{\alpha^{2}+\beta^{2}}\right) .
\end{aligned}
$$

Integration by parts in (23) gives a second integral form for $H(\alpha, \beta, t)$ :

$$
H(\alpha, \beta, t)=1-\mathrm{e}^{-\lambda t} \sqrt{\alpha^{2}+\beta^{2}} \int_{0}^{c t} J_{1}\left(\rho \sqrt{\alpha^{2}+\beta^{2}}\right) \exp \left(\frac{\lambda}{c} \sqrt{c^{2} t^{2}-\rho^{2}}\right) \mathrm{d} \rho .
$$

We can check that the integral representation (23) produces the series representation (22). By expanding the exponential factor in the integral in (23) and applying the Sonine formula (see, for instance, Bowman (1958, p. 97, Equation (6.43))), we obtain

$$
\begin{aligned}
\frac{\lambda \mathrm{e}^{-\lambda t}}{c} & \int_{0}^{c t} J_{0}\left(\rho \sqrt{\alpha^{2}+\beta^{2}}\right) \frac{\exp \left((\lambda / c) \sqrt{c^{2} t^{2}-\rho^{2}}\right)}{\sqrt{c^{2} t^{2}-\rho^{2}}} \rho \mathrm{d} \rho \\
& =\frac{\lambda \mathrm{e}^{-\lambda t}}{c} \int_{0}^{c t} \rho J_{0}\left(\rho \sqrt{\alpha^{2}+\beta^{2}}\right) \sum_{k=0}^{\infty}\left(\frac{\lambda}{c}\right)^{k} \frac{\left(\sqrt{c^{2} t^{2}-\rho^{2}}\right)^{k-1}}{k !} \mathrm{d} \rho \\
& =\mathrm{e}^{-\lambda t} \sum_{k=0}^{\infty}\left(\frac{\lambda}{c}\right)^{k+1} \frac{(c t)^{k+1}}{k !} \int_{0}^{1} w\left(1-w^{2}\right)^{(k-1) / 2} J_{0}\left(c t w \sqrt{\alpha^{2}+\beta^{2}}\right) \mathrm{d} w \\
& =\mathrm{e}^{-\lambda t} \sum_{k=0}^{\infty} \frac{(\lambda t)^{k+1} 2^{(k-1) / 2} \Gamma\left(\frac{1}{2}(k+1)\right)}{k !\left(c t \sqrt{\alpha^{2}+\beta^{2}}\right)^{(k+1) / 2}} J_{(k+1) / 2}\left(c t \sqrt{\alpha^{2}+\beta^{2}}\right) \\
& =\mathrm{e}^{-\lambda t} \sum_{n=1}^{\infty} \frac{(\lambda t)^{n}}{(n-1) !} \frac{2^{(n-2) / 2} \Gamma\left(\frac{1}{2} n\right)}{\left(c t \sqrt{\alpha^{2}+\beta^{2}}\right)^{n / 2}} J_{n / 2}\left(c t \sqrt{\alpha^{2}+\beta^{2}}\right) \\
& =\mathrm{e}^{-\lambda t} \sum_{n=1}^{\infty} \frac{(\lambda t)^{n}}{n !} \frac{2^{n / 2} \Gamma\left(\frac{1}{2} n+1\right)}{\left(c t \sqrt{\alpha^{2}+\beta^{2}}\right)^{n / 2}} J_{n / 2}\left(c t \sqrt{\alpha^{2}+\beta^{2}}\right) .
\end{aligned}
$$

By adding the second term of (23) we thus obtain the series representation (22) of the characteristic function $H(\alpha, \beta, t)$.

From both the series and integral representations (22) and (23) (and most easily from (24)), it immediately follows that the function $H(\alpha, \beta, t)$ satisfies the following initial conditions:

$$
\begin{gathered}
H(\alpha, \beta, 0)=1, \\
H_{t}(\alpha, \beta, 0)=0 .
\end{gathered}
$$


We can now establish a theorem concerning the equation governing the density (20).

Theorem 3. The density (20) of the absolutely continuous component of $(X(t), Y(t))$ is the fundamental solution to the two-dimensional telegraph equation

$$
\frac{\partial^{2} p}{\partial t^{2}}+2 \lambda \frac{\partial p}{\partial t}=c^{2}\left\{\frac{\partial^{2} p}{\partial x^{2}}+\frac{\partial^{2} p}{\partial y^{2}}\right\}
$$

(also called the planar damped wave equation).

Proof. By means of the exponential substitution

$$
p(x, y, t)=\mathrm{e}^{-\lambda t} w(x, y, t),
$$

(26) becomes

$$
\frac{\partial^{2} w}{\partial t^{2}}-c^{2}\left\{\frac{\partial^{2} w}{\partial x^{2}}+\frac{\partial^{2} w}{\partial y^{2}}\right\}=\lambda^{2} w
$$

By using the transformation

$$
\xi=\sqrt{c^{2} t^{2}-\left(x^{2}+y^{2}\right)}
$$

we can reduce the partial differential equation (28) to the ordinary differential equation

$$
\frac{\mathrm{d}^{2} \varphi}{\mathrm{d} \xi^{2}}+\frac{2}{\xi} \frac{\mathrm{d} \varphi}{\mathrm{d} \xi}-\frac{\lambda^{2}}{c^{2}} \varphi=0
$$

where

$$
\varphi(\xi)=w\left(\sqrt{c^{2} t^{2}-\left(x^{2}+y^{2}\right)}\right) .
$$

By introducing the new function $\psi(\xi)$, such that

$$
\varphi(\xi)=\xi^{-1} \psi(\xi)
$$

(30) can be further transformed into the linear ordinary differential equation

$$
\psi^{\prime \prime}-\frac{\lambda^{2}}{c^{2}} \psi=0,
$$

which has constant coefficients and whose general solution is

$$
\psi(\xi)=A \mathrm{e}^{(\lambda / c) \xi}+B \mathrm{e}^{-(\lambda / c) \xi}
$$

where $A$ and $B$ are arbitrary constants.

By taking into account all the substitutions carried out above, namely (31), (29) and (27), we obtain the following solution to (26):

$$
\begin{aligned}
u(x, y, t)= & \frac{\mathrm{e}^{-\lambda t}}{\sqrt{c^{2} t^{2}-\left(x^{2}+y^{2}\right)}} \\
& \times\left\{A \exp \left(\frac{\lambda}{c} \sqrt{c^{2} t^{2}-\left(x^{2}+y^{2}\right)}\right)+B \exp \left(-\frac{\lambda}{c} \sqrt{c^{2} t^{2}-\left(x^{2}+y^{2}\right)}\right)\right\} .
\end{aligned}
$$


For various constants $A$ and $B$, this forms some subspace in the solution space of the telegraph equation (26).

The density (20) can be obtained from (32) by setting $A=\lambda /(2 \pi c)$ and $B=0$. Thus, taking into account (25), we can conclude that the density (21) is the solution of the Cauchy problem

$$
\frac{\partial^{2} f}{\partial t^{2}}+2 \lambda \frac{\partial f}{\partial t}=c^{2}\left\{\frac{\partial^{2} f}{\partial x^{2}}+\frac{\partial^{2} f}{\partial y^{2}}\right\}, \quad f(x, y, 0)=\delta(x, y), \quad f_{t}(x, y, 0)=0,
$$

and, therefore, that its absolutely continuous component $p(x, y, t)$ is the fundamental solution to (26). The theorem is thus completely proved.

We now consider the limiting behaviour of the motion under the Kac condition (6). First, we note that, by dividing by $\lambda$ and passing to the limit under the condition (6), the telegraph equation (26) becomes the well-known planar heat equation. We now check that, under the same condition, the distribution (20) tends to the transition density of two-dimensional standard Brownian motion. To do so, it suffices to rewrite the density (20) in the form

$$
p(x, y, t)=\frac{\lambda}{2 \pi c^{2} t} \frac{\exp \left(-\lambda t+\lambda t \sqrt{1-\left(x^{2}+y^{2}\right) /\left(c^{2} t^{2}\right)}\right)}{\sqrt{1-\left(x^{2}+y^{2}\right) /\left(c^{2} t^{2}\right)}}
$$

and to expand the radical in the exponential factor using the well-known series

$$
\sqrt{1-z}=1-\frac{1}{2} z-\frac{1 \cdot 1}{2 \cdot 4} z^{2}-\frac{1 \cdot 1 \cdot 3}{2 \cdot 4 \cdot 6} z^{3}-\cdots,
$$

which converges absolutely for $|z|<1$. The application of this last formula is justified because

$$
z=\frac{x^{2}+y^{2}}{c^{2} t^{2}}<1 \quad \text { for any }(x, y) \in \operatorname{int} C_{c t} .
$$

Thus, as is easy to see, under the Kac condition (6), we have

$$
p(x, y, t)=\frac{\lambda}{2 \pi c^{2} t} \frac{\exp \left(-\lambda t+\lambda t \sqrt{1-\left(x^{2}+y^{2}\right) /\left(c^{2} t^{2}\right)}\right)}{\sqrt{1-\left(x^{2}+y^{2}\right) /\left(c^{2} t^{2}\right)}} \rightarrow \frac{1}{2 \pi t} \exp \left(-\frac{x^{2}+y^{2}}{2 t}\right),
$$

which is the transition density of two-dimensional standard Brownian motion.

This entirely accords with the similar limiting results obtained, using substantially different methods, by Pinsky (1976), Orsingher (2000), and Kolesnik (2001), (2003).

\section{Acknowledgements}

This paper was written while the first author was Visiting Professor of the Dipartimento di Statistica, Probabilità e Statistiche Applicate of the University of Rome 'La Sapienza'. He wishes to thank the University of Rome 'La Sapienza' for supporting this research and his co-author Professor Enzo Orsingher for his hospitality. The authors are grateful to Dr Lila Di Caprio for checking the English text and to Dr Eugene Kuznetsov for computer processing of the file. The authors wish to thank an anonymous referee for his helpful comments and, in particular, for bringing the papers by Stadje (1987), (1989) and Masoliver et al. (1993) to their attention. 


\section{References}

Bartlett, M. (1978). A note on random walks at constant speed. Adv. Appl. Prob. 10, 704-707.

Bowman, F. (1958). Introduction to Bessel Functions. Dover, New York.

Brooks, E. (1999). Probabilistic methods for a linear reaction-hyperbolic system with constant coefficients. Ann. Appl. Prob. 9, 719-731.

Di Crescenzo, A. (2002). Exact transient analysis of a planar motion with three directions. Stoch. Stoch. Reports 72, 175-189.

Goldstein, S. (1951). On diffusion by discontinuous movements and the telegraph equation. Quart. J. Mech. Appl. Math. 4, 129-156.

Gradshteyn, I. S. And Ryzhik, I. M. (1980). Tables of Integrals, Series and Products. Academic Press, New York.

KAC, M. (1974). A stochastic model related to the telegrapher's equation. Rocky Mountain J. Math. 4, 497-509.

KolesNiK, A. D. (1989). A model of Markovian random evolution on a plane. In Analytical Methods for Studying the Evolution of Stochastic Systems, Akad. Nauk. Ukrain. SSR, Inst. Math., Kiev, pp. 55-61 (in Russian).

KolesniK, A. D. (2001). Weak convergence of a planar random evolution to the Wiener process. J. Theoret. Prob. 14, 485-494.

KolesNiK, A. D. (2003). Weak convergence of the distributions of Markovian random evolutions in two and three dimensions. Bull. Acad. Sci. Moldova 3, 41-52.

KolesniK, A. D. AND Orsingher, E. (2002). Analysis of a finite-velocity planar random motion with reflection. Theory Prob. Appl. 46, 132-140.

Kolesnik, A. D. And Turbin, A. F. (1998). The equation of symmetric Markovian random evolution in a plane. Stoch. Process. Appl. 75, 67-87.

Leorato, S. and Orsingher, E. (2004). Bose-Einstein-type statistics, order statistics and planar random motions with three directions. Adv. Appl. Prob. 36, 937-970.

LeOrato, S., Orsingher, E. And ScAvino, M. (2003). An alternating motion with stops and the related planar, cyclic motion with four directions. Adv. Appl. Prob. 35, 1153-1168.

Masoliver, J., Porrà, J. M. ANd Weiss, G. H. (1993). Some two and three-dimensional persistent random walks. Physica A. 193, 469-482.

Orsingher, E. (1986). A planar random motion governed by the two-dimensional telegraph equation. J. Appl. Prob. 23, 385-397.

Orsingher, E. (1990). Probability law, flow function, maximum distribution of wave-governed random motions and their connections with Kirchoff's laws. Stoch. Process. Appl. 34, 49-66.

OrSINGHER, E. (2000). Exact joint distribution in a model of planar random motion. Stoch. Stoch. Reports 69, 1-10.

PINSKY, M. (1976). Isotropic transport process on a Riemannian manifold. Trans. Amer. Math. Soc. 218, 353-360.

PINSKy, M. (1991). Lectures on Random Evolution. World Scientific, River Edge, NJ.

Stadje, W. (1987). The exact probability distribution of a two-dimensional random walk. J. Statist. Phys. 46, $207-216$.

Stadje, W. (1989). Exact probability distributions for non-correlated random walk models. J. Statist. Phys. 56, 415-435.

Tolubinsky, E. V. (1969). The Theory of Transfer Processes. Naukova Dumka, Kiev. 